\title{
Millikan e a questão do potencial de contato no experimento do efeito fotoelétrico
}

\author{
Millikan and the contact potential question on the photoelectric effect experiment \\ Carlos Alberto dos Santos*1 \\ ${ }^{1}$ Universidade Federal Rural do Semiárido, Centro de Ciências Exatas e Naturais, Av. Francisco Mota, 572, 59.625-900, \\ Mossoró, RN, Brasil
}

Recebido em 25 de Outubro, 2017. Revisado em 15 de Dezembro, 2017. Aceito em 15 de Dezembro, 2017.

\begin{abstract}
Apresenta-se neste artigo o tratamento teórico e experimental dado por Millikan à questão do potencial de contato em seus experimentos sobre o efeito fotoelétrico. O prêmio Nobel concedido a Millikan em 1923, teve como motivação seus experimentos que resultaram na medida da carga do elétron (experimento da gota de óleo) e da constante de Planck (experimento sobre o efeito fotoelétrico). A precisão com que Millikan obteve a constante de Planck deve-se à importância que ele atribuiu ao potencial de contato entre dois metais eletricamente conectados. Ao apresentar seus cálculos para o potencial de contato em um dos artigos publicados em 1916, Millikan exibe um resultado sutilmente errado que aparentemente passou despercebido pelos estudiosos do assunto. Em 1921, Millikan corrige o equívoco sem mencionar o erro de 1916. Se este erro não resultou de má digitação, aparentemente não há justificativa plausível para que ele tenha ocorrido.
\end{abstract}

Palavras-chave: efeito fotoelétrico, potencial de contato, constante de Planck, Millikan.

It will be presented in this paper the theoretical and experimental Millikan's approach to the contact potential question in his experiments about the photoelectric effect. The 1923 Nobel prize won by Millikan, had as motivation his experiments which resulted on the electron charge (oil drop experiment) and Planck constant (photoelectric effect experiment) measurements. The precision with which Millikan obtained the Planck constant is due to the importance Millikan attributed to the contact potential between two electrically connected metals. Presenting his calculations on the contact potential in one of his 1916 papers, Millikan showed one result subtly wrong, apparently not perceived by the scholars of the subject. In 1921, Millikan corrected the erroneous equation without mention the 1916 mistake. If this mistake was not due to bad digitation, apparently there is no plausible justification for their occurency.

Keywords: photoelectric effect, contact potential, Planck constant, Millikan.

\section{Introdução}

O efeito fotoelétrico é um dos mais importantes marcos do desenvolvimento da física moderna [1-4]. Descoberto acidentalmente por Hertz, quando ele tentava produzir ondas eletromagnéticas em 1887 [3], o fenômeno desafiou a inteligência humana durante aproximadamente 18 anos, até ser decifrado em 1905 [5], quando Einstein propôs sua famosa equação, pela qual ganhou o Prêmio Nobel de Física de 1921 [6], conforme consta na página oficial do Prêmio Nobel

O Prêmio Nobel em Física de 1921 foi concedido a Albert Einstein "por suas contribuições à Física Teórica, e especialmente pela descoberta da lei do efeito fotoelétrico" (Tradução nossa).

*Endereço de correspondência: cas.ufrgs@gmail.com 1 https ://www.nobelprize.org/nobel_prizes/physics/ laureates/1921/
O que se entendia por "lei do efeito fotoelétrico" era simplesmente o que hoje costuma-se denominar "equação de Einstein para o efeito fotoelétrico". Como se verá mais abaixo, durante aproximadamente 20 anos a comunidade científica separou a equação de Einstein da sua hipótese sobre o quantum de luz.

A equação de Einstein para o efeito fotoelétrico é de uma simplicidade tão admirável, quanto complexa era a ideia que a sustentava. Alguns livros didáticos [7-9] costumam publicá-la da seguinte forma:

$$
e V_{0}=h \nu-\phi
$$

onde e é a carga do elétron, $\mathrm{V}_{0}$ é denominado potencial de corte, h é a constante de Planck, $\nu$ é a frequência da radiação incidente e $\phi$ é a função trabalho do material que emite os fotoelétrons. Existe uma certa confusão na literatura sobre o significado da função trabalho nessa equação, assim como em outras formas em que a eq. (1) é escrita. Uma discussão detalhada sobre essa questão pode ser encontrada em [3,10]. É suficiente aqui escrever uma 
outra forma da eq. (1), substituindo $\mathrm{eV}_{0}$ pela energia cinética máxima dos elétrons liberados pela superfície metálica. Insisto que essa substituição não é inteiramente correta, mas ela é cômoda para a discussão conceitual a seguir, e talvez por isso os livros didáticos a utilizem [7-9]. Então, a eq. (1) pode ser assim reescrita:

$$
E_{C i n M a x}=\left(\frac{1}{2} m v^{2}\right)_{\max }=h \nu-\phi,
$$

onde $\mathrm{m}$ é a massa do elétron e v é sua velocidade ao ser expelido pelo material.

O esquema do aparato experimental geralmente utilizado nos laboratórios didáticos para investigar a equação de Einstein [Eqs. (1) e (2)] é similar a este exibido na Fig. 1. Os eventos envolvendo essas equações e a Fig. 1 podem ser narrados de um modo que já utilizei em trabalho anterior [3]:

O efeito fotoelétrico começa com a incidência de uma radiação eletromagnética com frequência $\nu$, em determinada superfície metálica. Se a energia $h \nu$ da radiação for superior à energia de ligação de determinados elétrons aos átomos do material, esses elétrons serão liberados, mas até chegarem à superfície eles perdem energia proporcionalmente à profundidade de onde foram liberados do átomo. Existem vários mecanismos pelos quais se dá essa perda de energia, mas isso não vem ao caso aqui. O que importa aqui é saber que quanto mais distante da superfície, maior a perda de energia. Depois que chegam à superfície, esses elétrons têm que superar uma barreira superficial para serem lançados ao exterior. Essa barreira superficial se materializa

\section{Aparato para observação do efeito fotoelétrico}

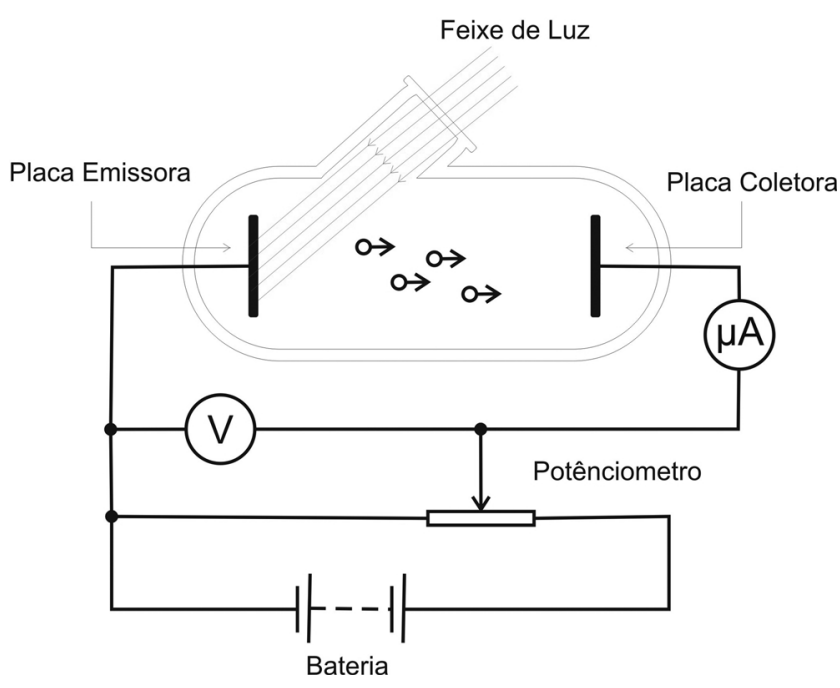

Figura 1: Esquema simplificado de aparato para observação do efeito fotoelétrico[11]. na forma de uma força eletromotriz de contato, ou simplesmente potencial de contato. Então, apenas os elétrons que são liberados dos átomos na superfície do material, têm a ver com $\phi$.

O texto acima baseia-se na hipótese levantada por Einstein ao apresentar sua equação para o efeito fotoelétrico [11]:

Quanta de energia penetram na camada superficial do material, e sua energia é transformada, pelo menos em parte, em energia cinética dos elétrons. A forma mais simples que se pode imaginar é que o quantum de luz transfere toda sua energia a um único elétron: assumiremos que é assim que ocorre. Todavia, não se deve excluir a possibilidade que apenas parte da energia dos elétrons venha do quantum de luz ${ }^{2}$

Essa hipótese marca o lançamento da ideia do quantum do luz, aquilo que 20 anos depois ficou conhecido como fóton. Einstein arremata sua hipótese fazendo a seguinte previsão a respeito da fórmula apresentada acima [11]:

Se a fórmula deduzida está correta, então $V_{0}$, quando representado em coordenadas cartesianas em função da frequência da luz incidente, deve ser uma linha reta cuja inclinação é independente da natureza da substância emissora.

Nessa história há duas questões. Uma é a hipótese da quantização da luz, que evoluiu para a hipótese da sua natureza corpuscular. Essa questão passou por um longo debate na comunidade científica, entre 1905 e 1925, e não será objeto de discussão aqui. Ela foi detalhadamente discutida em [3,12-16]. A outra questão diz respeito à validade da eq. (1) Embora Einstein tenha associado sua hipótese do quantum de luz à eq. (1), a comunidade científica dissociou esses dois eventos. Ou seja para muitos cientistas importantes no início do século 20, uma coisa era a equação de Einstein para o efeito fotoelétrico, e outra muito diferente era sua hipótese do quantum de luz. Essa história está muito bem discutida em [12,13], e não faz parte do escopo do presente trabalho, cujo objetivo é discutir os experimentos realizados por Millikan e seus contemporâneos para verificar a eq. (1).

Contrariando o que praticamente todos os textos didáticos dizem, os experimentos de Millikan não comprovaram a teoria de Einstein para o efeito fotoelétrico. Millikan e seus contemporâneos apenas validaram a equação de Einstein. Na verdade era apenas essa a intenção, ou

\footnotetext{
2 Tradução nossa, a partir do texto publicado no American Journal of Physics.

3 As eqs. 11 e $\sqrt{2}$ são equivalentes. Por simplicidade sempre me referirei à eq. 11, mas eventualmente usarei a energia cinética máxima dos elétrons na discussão, sem necessariamente me referir à eq. 2.
} 
seja o que eles queriam era determinar a dependência funcional correta para $\left(E_{\text {CinMax }}, \nu\right)$. Essa corrida pelos resultados experimentais se deu entre 1905 e 1916. Alguns pesquisadores mostraram a linearidade da equação, como deduzida por Einstein, mas outros encontraram relações não lineares, com diferentes expoentes na frequência da luz $[12,13,16]$.

Entre todos os estudiosos dedicados à questão, Robert Andrews Millikan foi sem dúvida o que mais se destacou. Passou mais de 10 anos realizando experimentos para mostrar que a equação de Einstein estava errada e terminou mostrando que ela não apenas estava correta, como também possibilitou a primeira obtenção experimental da constante de Planck. Um de seus trabalhos sobre o assunto, publicado em 1916 [17], foi um dos motivos para ele ganhar o Nobel de Física de 1923 [18]. A acuidade com que realizava seus experimentos, permitiu-lhe detectar vários problemas que levaram seus concorrentes a resultados equivocados. Entre as inovações exploradas por ele, destaca-se o tratamento dado à questão do potencial de contato, que se mostrou crucial para a precisão de seus resultados. Embora boa parte da literatura historiográfica atribua a paternidade dessa abordagem a Millikan, Karl Taylor Compton e Owen Willans Richardson talvez tenham sido os pioneiros na publicação de resultados experimentais sobre o assunto [19-22]. Apesar da importância histórica dessa constatação, sua análise está fora do escopo do presente trabalho.

Há um aspecto interessante na diferença do tratamento do potencial de contato entre Millikan e seus contemporâneos. Millikan percebeu que a equação de Einstein poderia ser usada para medidas precisas do potencial de contato entre diferentes metais.

Embora seja de inegável relevância, essa questão do potencial de contato não é considerada nos textos didáticos, nem na quase totalidade da literatura a respeito da história do efeito fotoelétrico. Ao meu conhecimento, a única exceção na literatura historiográfica é o trabalho de Allan Franklin [16]. Essa constatação é ainda mais notável quando se leva em conta um erro sutil cometido por Millikan em suas equações e que só foi por ele corrigido em 1921. Apesar dessa correção, todos os artigos de historiadores que eu li a respeito do trabalho de Millikan reproduzem sua equação com o erro. É sobre isso que tratarei no presente trabalho. Ou seja, de um lado apresentarei a abordagem experimental que Millikan fez do potencial de contato para medir com grande precisão a constante de Planck, e de outro lado apresentarei um erro sutil cometido por Millikan nos cálculos que ele elaborou para justificar o uso do potencial de contato.

\section{O experimento de Millikan para testar a equação de Einstein}

O último e mais famoso arranjo experimental utilizado por Millikan para investigar o efeito fotoelétrico é apresentado na Fig. 2. Pelo agradecimento que W.H. Kadesch

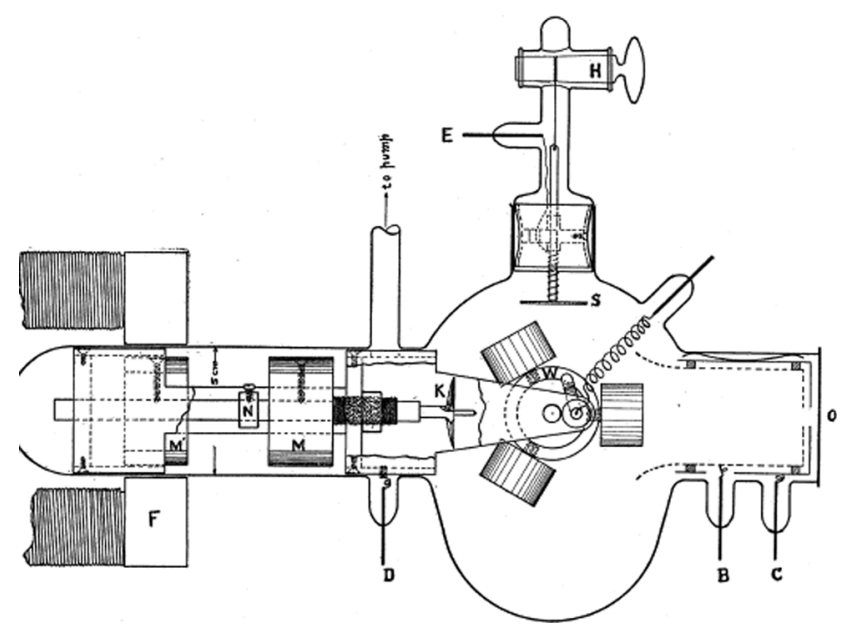

Figura 2: Esquema do equipamento usado por Millikan a partir de 1905 (Cortesia da APS Journals). Uma descrição moderna de como as medidas foram realizadas é apresentada em [27]

faz em seu trabalho sobre o equipamento [23], depreendese que Millikan o encarregou de desenvolver e testar o equipamento, permitindo-lhe que publicasse o trabalho sem sua participação Aparentemente esse era o padrão seguido por Millikan. Ou seja, ele costumava definir quais artigos seus colaboradores deveriam assinar, e quais ele assinaria. Algo similar aconteceu no caso do experimento da gota de óleo [24].

Kadesch concluiu a montagem do equipamento e obteve os primeiros resultados no início de janeiro de 1914 . O exemplar da Physical Review com seu trabalho foi publicado em maid ${ }^{4} \mathrm{Na}$ reunião da American Physical Society, em 24 de abril, Millikan apresenta provavelmente o primeiro trabalho em que ele menciona a expressão "direct determination of $h$ " [25], que será repetida no seu famoso artigo de 1916 [17]. Nessa reunião ele também discute pela primeira vez a questão do potencial de contato [26], algo que não foi objeto de análise no mencionado trabalho de Kadesch. Antes de continuar com a análise desses experimentos, vejamos alguns detalhes importantes do equipamento utilizado por Millikan.

A concepção desse equipamento e a metodologia experimental resultaram da criteriosa análise que Millikan fez das fontes de erro em seus experimentos anteriores e nos experimentos de seus contemporâneos, entre os quais destacam-se [3]:

1. A precisão na medida da fotocorrente é vital para a comprovação da equação de Einstein. Millikan percebeu que um filme de impureza na superfície do eletrodo produzia corrente centenas de vezes menores do que aquela produzida por um eletrodo limpo. A lâmina giratória $\mathrm{K}$ foi introduzida para providenciar a limpeza superficial dos eletrodos.

2. Um problema na detecção dos elétrons liberados, é que a corrente não vem exclusivamente do eletrodo. Elétrons produzidos por luz refletida, so-

${ }^{4}$ Conforme arquivo da APS: https://journals.aps.org/pr/issues/3/5. 
bretudo nas paredes do equipamento resultam em erros nas medidas. Essa situação é mais crítica quando a fotocorrente é fraca. Millikan resolveu esse problema usando radiação que não fosse capaz de liberar elétrons das paredes do equipamento. Isso foi possível por causa da apropriada seleção de catodos: lítio, sódio e potássio.

3. O potencial de contato no eletrodo foi outro problema tratado quase que exclusivamente por Millikan. Quando submetida a teste experimental, a equação de Einstein exige um cuidado sutil que muitos autores não levaram em consideração. A função trabalho na eq. 1 tem duas componentes. Uma é a energia para liberar o elétron do átomo, e a outra é a energia necessária para liberar o elétron da superfície do catodo. Essa última componente está relacionada com o potencial de contato. Resultados anteriores que não comprovaram a equação de Einstein foram obtidos sem levar em consideração a medida do potencial de contato.

4. O número de pontos experimentais é essencial para a precisão do ajuste da curva teórica. Alguns resultados anteriores foram obtidos com poucas frequências. Millikan escolheu lítio, sódio e potássio para ter várias frequências à disposição.

5. Um erro grave cometido pelos antecessores de Millikan, foi o uso da energia mais frequente dos fotoelétrons, quando a equação de Einstein exige que seja a energia máxima. Millikan resolveu esse problema usando fonte luminosa com alto grau de monocromaticidade.

Vejamos agora uma breve descrição do equipamento esquematizado na Fig. 2. Um sistema eletromecânico (F) permite que a manipulação das amostras seja feita sem a abertura da câmara. À direita, o tambor (W) contém suporte para três amostras 5 Millikan usou lítio, sódio e potássio. Para observar o efeito fotoelétrico, a radiação entra pelo orifício $(\mathrm{O})$ à direita. $\mathrm{O}$ servomecanismo posiciona a amostra à frente do feixe luminoso. A fotocorrente é medida pela captura de cargas elétricas no copo de Faraday conectado aos eletrodos (B) e (C). O copo de Faraday já era um dispositivo usual em medidas de corrente elétrica naquela época ${ }^{6}$

Entre as questões levantadas por Millikan e resumidas acima, vamos fixarmo-nos naquela que se refere ao potencial de contato.

\section{A importância do potencial de contato na medida da constante de Planck}

O primeiro trabalho sistemático de Millikan sobre o potencial de contato foi concluído em setembro de 1915 ,

\footnotetext{
5 Esse artifício tecnológico é banal hoje em dia, mas, ao que sei essa foi a primeira vez que foi utilizado na pesquisa em física. ${ }^{6}$ https://en.wikipedia.org/wiki/Faraday_cup
}

e publicado em janeiro de 1916 na Physical Review [27]7 com título Einstein's photoelectric equation and contact electromotive force. Trata-se de um texto bem ao estilo do autor, com uma precisa e didática narrativa. No início do artigo, Millikan escreve a equação de Einstein,

$$
1 / 2 m v^{2}=V e=h \nu-p,
$$

e afirma que ela não pode ser vista sob qualquer fundamentação teórica satisfatória, e que portanto suas credenciais são puramente empíricas. No contexto do presente trabalho esse comentário de Millikan é irrelevante. O que interessa aqui são seus procedimentos para a investigação da validade da equação. Assim como Millikan, não entrarei em detalhes a respeito do que seja potencial de contato. Para uma revisão sugiro esses artigos [28-31]. Para quem compreende francês, recomendo o trabalho original de Alessandro Volta [32].

A quantidade p na eq. (3) tem duas componentes: (a) $\mathrm{p}_{1}$, que representa o trabalho necessário para liberar o elétron do átomo ao qual ele estava ligado, tornandoo um elétron livre no metal; (b) $\mathrm{p}_{2}$, que representa o trabalho necessário para que este elétron livre vença a barreira superficial do metal e fique disponível no meioambiente. Para aprofundar esta análise, observemos a Fig. 1. Além da radiação incidente, os elementos mais importantes dessa montagem são os dois eletrodos, denominados placa emissora e placa coletora. A luz incide sobre a placa emissora para liberar elétrons que serão coletados na placa coletora. Essas duas placas estão conectadas por meio do restante do circuito. Então, sem incidência de luz, e sem aplicação de voltagem, o que se tem na Fig. 1 são duas placas metálicas conectadas. O que acontece se essas duas placas são confeccionadas de materiais diferentes?

No final do século 18, Alessandro Volta mostrou que quando dois metais diferentes são colocados em contato, surge uma diferença de potencial (ddp) que só depende dos dois metais, sendo portanto independente dos materiais que os conectam. Atualmente essa diferença de potencial é conhecida como potencial de contato, mas já foi denominada potencial de Volta. Millikan a denomina força eletromotriz de contato (Contact E.M.F.). Para simplificar, a denominarei de PC (potencial de contato).

Então, na montagem de um experimento sobre o efeito fotoelétrico, depois que o elétron se livrar do átomo no interior do metal, ele terá que vencer a barreira do potencial de contato do par (placa emissora, placa coletora) para fazer parte da fotocorrente. Ou seja, é importante conhecer o PC do par de eletrodos.

Se há uma ddp entre os dois metais, haverá circulação de elétrons de um metal para o outro. Pergunta natural: quem perde e quem ganha elétrons? Ou seja, de qual metal sai o elétron na direção do outro metal? Para discutir corretamente essa questão, é necessário levar em conta o conceito de nível de Fermi [30], que obviamente

\footnotetext{
7 Conforme o arquivo da APS: https://journals.aps.org/pr/ issues/7/1
} 
ainda não existia na época dos experimentos de Millikan. Tentarei acompanhar a discussão de Millikan sem recorrer à ideia do nível de Fermi. Na sua discussão, Millikan usou zinco e cobre, e afirmou que elétrons fluem do zinco para o cobre. Por que isso é assim? Porque o cobre é mais eletronegativo do que o zincd 8 . Portanto, o potencial do cobre $\left(\mathrm{V}_{\mathrm{Cu}}\right)$ é superior ao do zinco $\left(\mathrm{V}_{\mathrm{Zn}}\right)$, e a ddp entre eles, ou PC deve ser:

$$
P C=V_{C u}-V_{Z n}
$$

Em termos dos trabalhos $\mathrm{p}_{2}$, essa expressão fica assim:

$$
P C=\frac{\left(p_{2}\right)_{C u}-\left(p_{2}\right)_{Z n}}{e}
$$

Em seus cálculos, Millikan usa $\left(p_{2}\right)_{Z n}=p_{2}$ e $\left(p_{2}\right)_{C u}=$ $p_{2}^{\prime}$. Daqui em diante seguirei a notação de Millikan para facilitar a comparação com seu artigo [27].

\section{Cálculo do PC apresentado por Millikan em 1916 e em 1921}

Como já foi dito, Millikan inicia seu artigo de 1916 com a eq.(3). Depois de uma série de discussões em torno de resultados obtidos por outros pesquisadores e da questão da energia máxima dos fotoelétrons, ele apresenta alguns dos seus resultados e introduz as componentes $\mathrm{p}_{1}$ e $\mathrm{p}_{2}$, com as definições que apresentei acima. Na seção 3 do artigo, intitulada The relation of contact E.M.F. and Einstein's equation, ele afirma que (tradução nossa):

a intersecção da reta [que representa a eq. de Einstein] com o eixo das frequências é a frequência a partir da qual o metal torna-se fotossensível. Para verificar isso, foi necessário deslocar na direção dos potenciais positivos, a reta $\mathrm{V}$ versus frequência por uma quantidade igual ao potencial de contato.

Essa frequência à qual Millikan se refere é denominada de frequência de corte. Ou seja, abaixo dessa frequência não há efeito fotoelétrico. Exatamente nessa frequência, o elétron é liberado com energia cinética nula. Então, fazendo $\mathrm{v}=0$ na eq. (3), obtém-se

$$
p=h \nu_{0},
$$

Foi a partir desse artifício que Millikan verificou com tanta precisão a equação de Einstein. A Fig. 3 é uma adaptação que fiz da figura apresentada por Millikan na famosa publicação de 1916. Veja que a reta experimental é deslocada para cima pela adição do potencial de contato. Na sequência Millikan apresenta seus cálculos para justificar o artifício.

\footnotetext{
8 Usando o conceito de nível de Fermi, ou energia de Fermi, o elétron flui do zinco para o cobre, porque este tem energia de Fermi $(7,00 \mathrm{eV})$ menor do que o zinco $(9,47 \mathrm{eV})$.
}

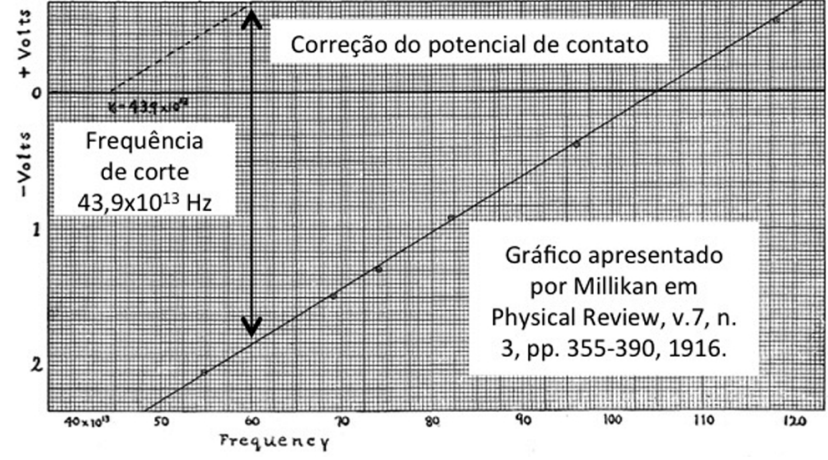

Figura 3: Resultados de Millikan, publicados em seu famoso artigo "A direct photoelectric determination of Planck's ' $h$ ' [18] (Cortesia da APS Journals).

Em sua eq. (4), aqui reproduzida como eq. (7), Millikan define o potencial de contato para o par zinco-cobre:

$$
\text { Contact E.M.F. }=\frac{p_{2}-p_{2}^{\prime}}{e},
$$

onde $\mathrm{p}_{2}$ refere-se ao zinco e $\mathrm{p}_{2}$ ' ao cobre.

Aplicando a eq. (6) para o zinco e o cobre, obtém-se

$$
h \nu_{0}=p=p_{1}+p_{2}
$$

e

$$
h \nu_{0}^{\prime}=p^{\prime}=p_{1}^{\prime}+p_{2}^{\prime}
$$

Subtraindo a segunda equação da primeira, obtém-se

$$
h \nu_{0}-h \nu_{0}^{\prime}=\left(p_{1}-p_{1}^{\prime}\right)+\left(p_{2}-p_{2}^{\prime}\right) .
$$

Substituindo $\left(p_{2}-p_{2}^{\prime}\right)$ em 77 , obtém-se

$$
\text { Contact E.M.F. }=\frac{h \nu_{0}-h \nu_{0}^{\prime}-\left(p_{1}-p_{1}^{\prime}\right)}{e} .
$$

Como foi dito acima, um dos objetivos de Millikan nesse trabalho era mostrar que a equação de Einstein possibilitava medidas precisas de potencial de contato entre diferentes metais. Então, ele analisou o experimento do efeito fotoelétrico a partir da utilização de dois metais diferentes como emissores e de um copo de Faraday como coletor.

A essa altura, convém colocar lado a lado os cálculos apresentados por Millikan em 1916 e 1921. As equações (10a) e (10b) são modificações da eq. (3) que Millikan introduziu para incluir o potencial de contato. Observe que em 1921 ele deixou de usar a letra $\mathrm{V}$ para a diferença de potencial e passou a usar PD (potential difference).

Em 1916 [eq. (10a]] e 1921 [eq. (10b]), Millikan escreveu:

$$
\begin{gathered}
1 / 2^{m v^{2}}=\left(V_{0}+K\right) e=h \nu-p=h \nu-h \nu_{0} \\
1 / 2 m v^{2}=\left(P D_{A}+K_{A}\right) e=h \nu-w_{A}=h \nu-h \nu_{0_{A}}
\end{gathered}
$$

Escrevendo as equs. (10) para outro metal tem-se: 


$$
\begin{gathered}
1 / 2^{m} v^{2}=\left(V_{0}^{\prime}+K^{\prime}\right) e=h \nu-p^{\prime}=h \nu-h \nu_{0}^{\prime} \\
1 / 2^{m} v^{2}=\left(P D_{B}+K_{B}\right) e=h \nu-w_{B}=h \nu-h \nu_{0_{B}}
\end{gathered}
$$

No artigo de 1916, Millikan subtraiu (10a) de 11a $\left[\left(V_{0}^{\prime}-V_{0}\right) e+\left(K^{\prime}-K\right) e=h \nu_{0}-h \nu_{0}^{\prime}\right]$, ao passo que em 1921 ele inverteu a subtração, ou seja subtraiu $111 \mathrm{~b}$ de (10b) $\left[\left(P D_{A}-P D_{B}\right) e+\left(K_{A}-K_{B}\right) e=h\left(\nu_{0_{B}}-\nu_{0_{A}}\right)\right]$.

Em 1916, Millikan definiu K'-K [eq. 112a] como o potencial de contato, ao passo que em 1921 o potencial de contato foi definido como $\mathrm{K}_{\mathrm{A}}-\mathrm{K}_{\mathrm{B}}$ [eq. [12b]]. Fazendo as devidas substituições, obtém-se:

$$
\begin{gathered}
\text { Contact E.M.F. }=\frac{h}{e}\left(\nu_{0}-\nu_{0}^{\prime}\right)-\left(V_{0}^{\prime}-V_{0}\right) \\
\text { Contact E.M.F. }=\frac{h}{e}\left(\nu_{0_{B}}-\nu_{0_{A}}\right)-\left(P D_{A}-P D_{B}\right)
\end{gathered}
$$

No artigo de 1916, a equação de Millikan correspondente à eq. 12a foi escrita com um sinal trocado, ou seja aparece no artigo a seguinte expressão para o potencial de contato entre os dois metais emissores:

$$
\text { Contact E.M.F. }=\frac{h}{e}\left(\nu_{0}-\nu_{0}^{\prime}\right)-\left(V_{0}-V_{0}^{\prime}\right) \text {. }
$$

A eq. 12b está correta, ao passo que a eq. 113 está errada. No entanto, Millikan e os autores que o citam, referem-se unicamente à eq. (13). Se a impressão de $\mathrm{V}_{0}-\mathrm{V}_{0}$ ' em vez de $\mathrm{V}_{0}$ '- $\mathrm{V}_{0}$ não foi um erro de digitação, não faço ideia do que tenha levado Millikan a fazer essa inversão. Veja que o fato de subtrair a eq. (10) da eq. (11) ou o inverso, não produz qualquer erro no resultado, desde que a definição do potencial de contato seja consistente. Ou seja, usando a notação que Millikan usou em 1921, subtrair a eq. (10) da eq. (11) exige que o potencial de contato seja definido como $\mathrm{K}_{\mathrm{B}}-\mathrm{K}_{\mathrm{A}}$, enquanto a outra subtração exige que o potencial de contato seja $K_{A}-K_{B}$. Isso pode ser visto de outro modo. Suponha que em 1921 Millikan tivesse feito a mesma subtração [(11)-(10)] que fez em 1916, mas definido o potencial de contato como $\mathrm{K}_{\mathrm{B}}-\mathrm{K}_{\mathrm{A}}$, em vez de $\mathrm{K}_{\mathrm{A}}-\mathrm{K}_{\mathrm{B}}$. Ele obteria a seguinte expressão para o potencial de contato:

$$
\text { Contact E.M.F. }=\frac{h}{e}\left(\nu_{0_{A}}-\nu_{0_{B}}\right)-\left(P D_{B}-P D_{A}\right)
$$

Veja que as equações (12a, 12b e 14 são formalmente idênticas. Elas diferem por um sinal no valor do potencial de contato, que é natural, pois ele vem do fato de que um metal é mais eletronegativo do que o outro. Por exemplo, no caso $\mathrm{Zn} / \mathrm{Cu}$, utilizado por Millikan, os elétrons fluem do zinco para o cobre porque este é mais eletronegativo, portanto tem um potencial maior, de modo que $\mathrm{K}_{\mathrm{Cu}}-\mathrm{K}_{\mathrm{Zn}}>0$ e $\mathrm{K}_{\mathrm{Zn}}-\mathrm{K}_{\mathrm{Cu}}<0$.

\section{Epílogo}

O escopo do presente artigo foi limitado à discussão de um erro sutil cometido por Millikan em um de seus artigos de 1916 [27], que aparentemente passou despercebido pelos estudiosos do assunto. Em 1921, Millikan corrigiu o erro, sem fazer menção ao mesmo [33]. Tudo indica que os autores desconsideraram a correção de 1921 e cristalizaram a expressão equivocada de 1916. Em artigo recente, Franklin faz um extenso estudo do experimento de Millikan e reproduz a equação de 1916 sem fazer qualquer referencia à correção de 1921 [16].

Pelos resultados publicados, fica claro que se o erro de sinal na fórmula não foi originado em má digitação, ele foi corrigido por Millikan durante a análise de seus experimentos. Todavia, chama a atenção o fato de Millikan não ter feito qualquer referência a essa situação em seu trabalho de 1921.

Quer tenha havido erro na manipulação algébrica, ou durante a digitação da equação, o fato histórico e pedagógico relevante em torno dessa questão do potencial de contato é que ela é desconsiderada pela literatura didática, como atestam $[10,28,34]$. O fato de o potencial de contato não ser levado em conta em textos didáticos implica na preservação de equívocos sérios em relação à verificação experimental da equação de Einstein, bem como à obtenção da constante de Planck. Por exemplo, os mais populares textos didáticos sugerem que a energia máxima dos elétrons no efeito fotoelétrico pode ser obtida a partir da medida do potencial de corte, ignorando a importância do potencial de contato. Além disso, o potencial de contato tem sido um tema de pesquisa vibrante desde o advento da física da matéria condensada, no final dos anos 1920 e início dos anos 1930 [31,35,36]. Portanto seu tratamento na formação inicial de professores de física, bem como na formação de bacharéis em física cumpre uma finalidade histórica e formativa.

\section{Agradecimento}

Agradeço ao professor Luiz Fernando Ziebell (IF-UFRGS) pela cuidadosa revisão do manuscrito.

\section{Referências}

[1] C.A. dos Santos, Ciência Hoje Online (2015), disponível em http://www.cienciahoje.org.br/noticia/v/ler/ id/2924/n/o_fisico_e_o_foton, acesso em 10.10.2017.

[2] C.A. dos Santos, Ciência Hoje 339, 61 (2016).

[3] C.A. dos Santos, in A Luz E Algumas Suas Tecnol., edited by C.A. dos Santos (Editora da UEPG, Ponta Grossa, 2017), p. 95-156.

[4] R. de A. Martins e P.S. Rosa, História Da Teoria Quântica: A Dualidade Onda-Partícula, de Einstein a De Broglie (Livraria da Física, São Paulo, 2014).

[5] B.R. Wheaton, Hist. Stud. Phys. Sci. 9, 299 (1978).

[6] R.H. Stuewer, Acta Phys. Pol. B 37, 543 (2006). 
[7] D. Halliday and R. Resnick, Física Básica - Vol. 4 (Livros Técnicos e Científicos, Rio de Janeiro, 1991).

[8] P.A. Tipler, Física Moderna (Guanabara Dois, Rio de Janeiro, 1981).

[9] R. Eisberg e R. Resnick, Física Quântica: Átomos, Moléculas, Sólidos, Núcleos E Partículas (Campus, Rio de Janeiro, 1986).

[10] J. Rudnick and D.S. Tannhauser, Am. J. Phys. 44, 796 (1976).

[11] A. Einstein, Am. J. Phys. 33, 367 (1965).

[12] R.H. Stuewer, in Hist. Philos. Perspect. Sci., edited by R.H. Stuewer (University of Minnesota Press, Minnesota, 1970), p. 246-263.

[13] H. Kragh, Sci. Educ. 1, 349 (1992).

[14] G. Holton, 1, 231 (1999).

[15] M. Niaz, S. Klassen, B. McMillan, and D. Metz, Sci. Stud. Sci. Educ. 94, 903 (2010).

[16] A. Franklin, Eur. J. Phys. 38, 573 (2013).

[17] R.A. Millikan, Phys. Rev. 7, 355 (1916).

[18] R.A. Millikan, Nobel Lect. 54 (1924), disponível em https://www.nobelprize.org/nobel_prizes/ physics/laureates/1923/millikan-lecture.pdf acesso em 20.09.2017

[19] O.W. Richardson and K.T. Compton, Science 35, 783 (1912).

[20] K.T. Compton, Philos. Mag. 23, 579 (1912).

[21] O.W. Richardson and K.T. Compton, Philos. Mag. 24, 575 (1912).

[22] O.W. Richardson, Phys. Rev. 4, 522 (1914).

[23] W.H. Kadesch, Phys. Rev. 3, 367 (1914).

[24] C.A. Santos, Rev. Bras. Ensino Física 17, 107 (1995).

[25] R.A. Millikan, Phys. Rev. 4, 73 (1914).

[26] R.A. Millikan and W.H. Souder, Phys. Rev. 4, 73 (1914).

[27] R.A. Millikan, Phys. Rev. 7, 18 (1916).

[28] D.R. Lloyd, Am. J. Phys. 83, 765 (2015).

[29] J.C. Riviere, Proc. Phys. Soc. Sect. B 70, 676 (1957).

[30] O. Vilitis, M. Rutkis, J. Busenberg, and D. Merkulov, Latv. J. Phys. Tech. Sci. 2, 48 (2016).

[31] S. Halas, Mater. Sci. 24, 951 (2006).

[32] A. Volta, Phil. Trans. R. Soc. L. 90, 403 (1800).

[33] R.A. Millikan, Phys. Rev. 18, 236 (1921).

[34] S. Klassen, Sci. Educ. 20, 719 (2011).

[35] L.A. DuBridge, Phys. Rev. 43, 727 (1933).

[36] N. Bjerrum, Acta Chem. Scand. 12, 945 (1958). 